\title{
ACCELERATION OF DIELS-ALDER REACTIONS BY REMOTE METHYL GROUPS
}

\author{
Janusz Baran' and Herbert Mayr* \\ Institut für Chemie der Medizinischen Universität zu Lü̈beck \\ Ratzeburger Allee 160, D-2400 Lubeck 1, Federal Republic of Germany \\ (Received in Germany 6 February 1989)
}

\begin{abstract}
The hexamethyl substituted bismethylenecyclopentane $1 a$ is 4 to 7 times more reactive towards the acetylenic dienophiles 2 and 3 than the nonmethylated 1,2-bismethylenecyclopentane 1b. This unusual consequence of branching is explained in terms of steric and electronic effects.
\end{abstract}

Substituent effects of alkyl groups depend on the degree of branching. While the electronic effects of branching are commonly small in the condensed phase, significant steric effects may be encountered. ${ }^{2}$ Branching usually accelerates reactions, in which bonds are broken in the rate determining step, e.g. homolytic ${ }^{3}$ or heterolytic ${ }^{4} \mathrm{CC}$ and $\mathrm{CX}$ bond fissions. On the other hand, bond forming reactions, e.g. cycloadditions ${ }^{5}$ or additions of radicals to alkenes ${ }^{6}$, often experience considerable retardations with increasing bulk of the alkyl substituents. We now report the unusual observation that a Diels-Alder reaction is accelerated as the bulkiness of one of the cycloaddends is increased.

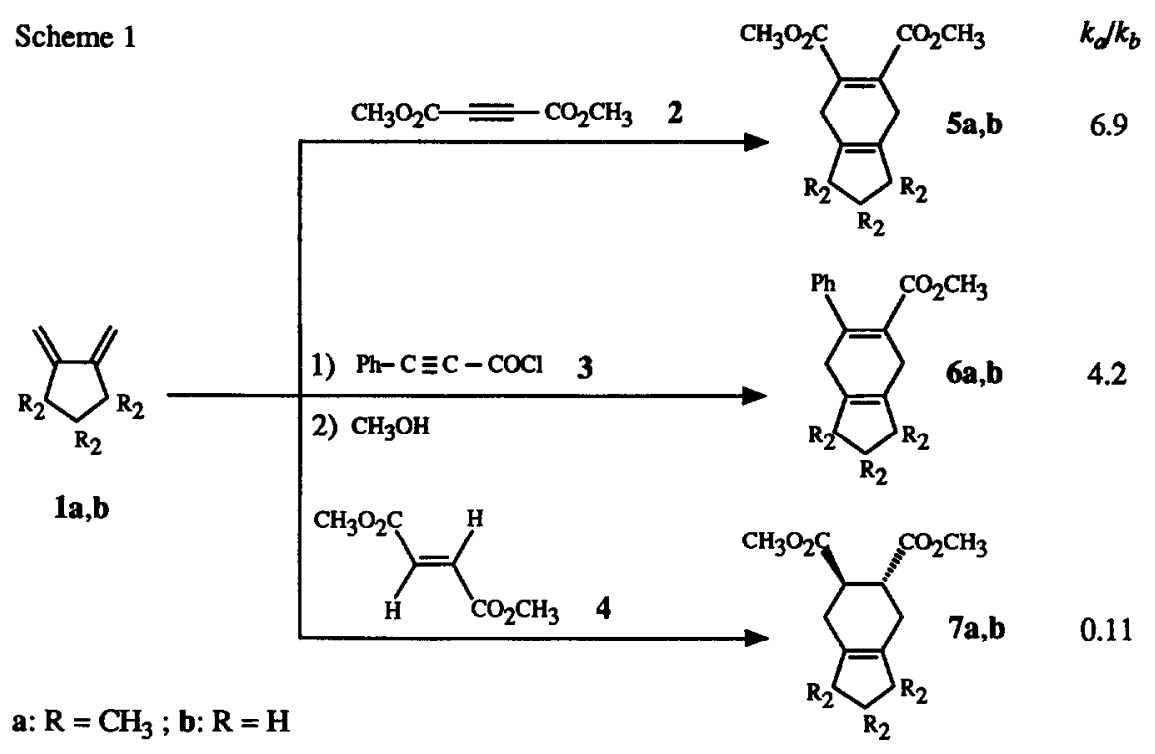

1,1,2,2,3,3-Hexamethyl-4,5-bis(methylene)cyclopentane 1a, ${ }^{7}$ an s-cis fixed 1,3-diene has been shown to undergo some unprecedented 1,4-additions, ${ }^{8}$ since its 1,2-reactivity is reduced by the bulky substituents attached to the 2and 3-position of the diene fragment. In order to use 1a as a mechanistic probe for the study of cycloaddition reactions, we needed information about the influence of the methyl groups on the 1,4-reactivity of la and we have, therefore, compared the reactivity of $1 \mathrm{a}$ and $\mathbf{1 b}$ towards the dienophiles $2-4$. 
While the acetylenic dicarboxylate 2 reacted smoothly with the dienes $1 \mathrm{a}$ and $1 \mathbf{b}$ at room temperature, the cycloaddition of 1a,b with the methyl ester of phenylpropiolic acid was very slow, even at $60^{\circ} \mathrm{C}$. As expected, ${ }^{9}$ the corresponding acid chloride 3 was considerably more reactive, and at room temperature gave cycloadducts, which were solvolyzed in methanol to afford $6 \mathrm{a}$ and $6 \mathrm{~b}$. The cycloadditions of 4 were sluggish at room temperature, but proceeded quantitatively in toluene within a few hours at 60 or $80^{\circ} \mathrm{C}$.

Competition experiments revealed that the acetylenic dienophiles 2 and 3 , in contrast to the ethylenic dienophile 4. react noticeably faster with the highly substituted diene $1 \mathrm{a}$ than with the nonmethylated homolog $1 \mathrm{~b}$ (right column of Scheme 1). How can $k_{d} / k_{b}$ ratios greater than 1 be explained? Force field calculations ${ }^{10}$ reveal that the reaction of $1 \mathrm{a}$ with acetylene is $0.4 \mathrm{kcal} / \mathrm{mol}$ more exothermic than the corresponding reaction of the nonmethylated compound $1 \mathrm{~b}$. The strain associated with the vicinity of 3 quaternary carbon centres in $1 \mathrm{a}$ is somewhat released when the $\mathrm{C}-1 / \mathrm{C}-2$ bond becomes shortened during the cycloaddition.

Scheme 2

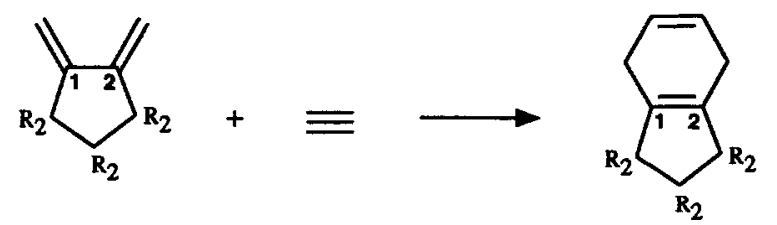

a) $\mathrm{R}=\mathrm{CH}_{3} ; \Delta E_{s}(\mathrm{MMX})=-1.76 \mathrm{kcal} / \mathrm{mol}$

b) $\mathrm{R}=\mathrm{H} ; \Delta E_{s}(\mathrm{MMX})=-1.35 \mathrm{kcal} / \mathrm{mol}$

If the difference of the reaction enthalpies is represented correctly by the molecular mechanics calculations, a $k_{a} / k_{b}$ value of 2 might be due to steric effects, if the geometrical reorganisation were complete in the transition state. Since Diels-Alder reactions are assumed to proceed through early transition states, the weight of the steric effect should be even smaller, and electronic contributions have to be considered. The ionization potential of $1 \mathrm{a}$ $\left(I P_{\mathrm{y}}(I)=8.4 \mathrm{eV}\right)^{11}$ was found to be $0.3 \mathrm{eV}$ lower than that of the nonmethylated compound $1 \mathrm{~b}\left(I P_{\mathrm{v}}(I)=8.73\right.$ $\mathrm{eV})^{12}$. Since all these reactions can be assumed to be controlled by the HOMO(diene)-LUMO(dienophile) interactions, ${ }^{13}$ the higher reactivity of $1 \mathrm{a}$ can be explained by the reduced HOMO-LUMO gap. We, therefore, suggest that a combination of steric and electronic effects accounts for the reactivity preference of $1 \mathrm{a}$ over $\mathbf{1 b}$ towards acetylenic dienophiles.

According to force field calculations (MMX), the Diels-Alder reaction of ethylene with 1a is also slightly more exothermic than the corresponding reaction with $1 \mathrm{~b}\left(\Delta \Delta H^{\mathrm{o}}=0.6 \mathrm{kcal} / \mathrm{mol}\right)$. Since the frontier orbital argument applies in the same way as above, the inverse reactivity ratio of $1 \mathrm{a}$ and $1 \mathrm{~b}$ towards fumarate 4 must be due to steric repulsions in the transition state $8 \mathbf{a}$.

\section{EXPERIMENTAL.}

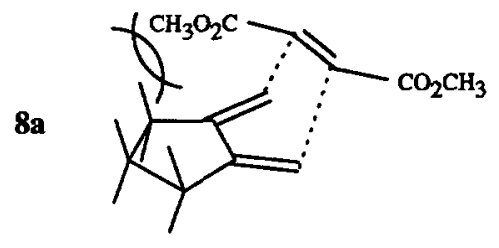

General. IR spectra were recorded on a Shimadzu IR-435 spectrometer. NMR spectra were taken on a Varian XI 200 spectrometer using tetramethylsilane as internal standard. Mass spectra were recorded on a 70-250E VG spectrometer and the microanalyses were carried out by Ilse Beetz, Microanalytisches Laboratorium, D-8640 
Kronach. Melting points are uncorrected. GC analyses were run on a Shimadzu GC 9A Gas Chromatograph equipped with FID.

3,4-Bis(methoxycarbonyl)-7,7,8,8,9,9-hexamethyl-bicyclo[4.3.0]nona-1(6),3-diene (5a). Compounds la (0.152 $\mathrm{g}, 0.852 \mathrm{mmol})$ and $2(0.115 \mathrm{~g}, 0.809 \mathrm{mmol})$ were mixed in absence of a solvent. An exothermic reaction took place, and the ${ }^{1} \mathrm{H}$ NMR spectrum taken after $5 \mathrm{~h}$ showed quantitative formation of $5 \mathrm{a}$, which was recrystallized from methanol. Mp 78-79 $\mathrm{C}$ - IR (KBI): 2971, 2939, 2909, 2863, 1727, 1437, 1300, 1271, 1228, $1060 \mathrm{~cm}^{-1}$. - ${ }^{1} \mathrm{H}$ $\operatorname{NMR}\left(\mathrm{CDCl}_{3}\right) \delta 0.81(\mathrm{~s}, 6 \mathrm{H}), 0.92(\mathrm{~s}, 12 \mathrm{H}), 2.92(\mathrm{~s}, 4 \mathrm{H}), 3.80(\mathrm{~s}, 6 \mathrm{H}),-{ }^{13} \mathrm{C} \mathrm{NMR}\left(\mathrm{CDCl}_{3}\right) \delta 21.45(\mathrm{q}), 23.63$ (q, double int.), 25.75 (t), 47.03 (s), 48.92 (s, double int.), 52.25 (q), 133.59 (s), 133.87 (s), 168.96 (s). - MS (70 eV) $\mathrm{m} / \mathrm{z}=320\left(\mathrm{M}^{+}, 19\right), 305(31), 289(20), 288(28), 273(100), 204(73), 177$ (15), 139 (59), 41 (14). - Anal. Calcd for $\mathrm{C}_{19} \mathrm{H}_{28} \mathrm{O}_{4}$ (320.4): C, 71.22; $\mathrm{H}, 8.81$. Found: $\mathrm{C}, 70.90 ; \mathrm{H}, 8.84$.

3,4-Bis(methoxycarbonyl)-bicyclo[4.3.0]nona-1(6),3-diene (5b). Compounds $1 \mathrm{~b}(0.113 \mathrm{~g}, 1.20 \mathrm{mmol})$ and 2 $(0.162 \mathrm{~g}, 1.14 \mathrm{mmol})$ were combined without a solvent to give $5 \mathrm{~b}$ quantitatively $\left({ }^{1} \mathrm{H}\right.$ NMR spectrum) in an exothermic reaction. Five hours after mixing the reactants, the product was recrystallized from methanol. Mp $40-41^{\circ} \mathrm{C}$ - IR (KBr): 2946, 2881, 2838, 2805, 1729, 1718, 1703, 1643, 1432, 1322, 1265, 1261, 1190, 1161, 1077 , $1051,1007,936,765 \mathrm{~cm}^{-1} .{ }^{1} \mathrm{H}$ NMR $\left(\mathrm{CDCl}_{3}\right) \delta 1.90$ (br. quint, $J=7 \mathrm{~Hz}, 2 \mathrm{H}$ ), 2.30 (br.t, $\left.J=7 \mathrm{~Hz}, 4 \mathrm{H}\right), 2.98$ (br. s, $4 \mathrm{H}), 3.79(\mathrm{~s}, 6 \mathrm{H}) .{ }^{13} \mathrm{C}$ NMR $\left(\mathrm{CDCl}_{3}\right) \delta 21.73(\mathrm{t}), 29.26(\mathrm{t}), 34.87(\mathrm{t}), 52.23(\mathrm{q}), 130.33(\mathrm{~s}), 133.46(\mathrm{~s})$,

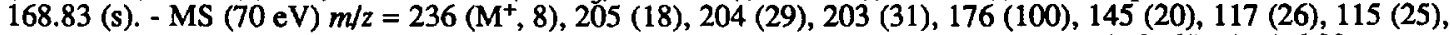
105 (23), 91 (20), 59 (18). - Anal. Calcd for $\mathrm{C}_{13} \mathrm{H}_{16} \mathrm{O}_{4}$ (236.3): C, 66.09; $\mathrm{H}, 6.83$. Found: C, 65.75; $\mathrm{H}, 6.82$.

3-Methoxycarbonyl-7,7,8,8,9,9-hexamethyl-4-phenyl-bicyclo[4.3.0]nona-1(6),3-diene (6a). Diene 1a (0.218 g, $1.22 \mathrm{mmol})$ and $3(0.103 \mathrm{~g}, 0.626 \mathrm{mmol})$ were combined and kept for $3 \mathrm{~d}$ at ambient temperature. The mixture was dissolved in $2 \mathrm{~mL}$ of dry ether and added dropwise to the stirred suspension of $\mathrm{NaHCO}_{3}(0.12 \mathrm{~g})$ in methanol $(3 \mathrm{~mL})$. After $1 \mathrm{~h}$, the solvents were evaporated under reduced pressure, and the dry residue was extracted with two 5-mL portions of ether. For this purpose, ether was added, stirred with a spatula, put into an ultrasonic bath for $1 \mathrm{~min}$, and filtered. The ether was evaporated and the residue was recrystallized from methanol to give colorless crystals $(0.173 \mathrm{~g}, 82 \%)$ with $\mathrm{mp} 116-117^{\circ} \mathrm{C}$ - - $\mathbb{R}(\mathrm{KBr}): 2968,2939,2904,2861,1711,1698,1442$, $1431,1373,1306,1247,1054,766,701 \mathrm{~cm}^{-1}$. ${ }^{1}{ }^{\mathrm{H}} \mathrm{NMR}\left(\mathrm{CDCl}_{3}\right) \delta 0.84(\mathrm{~s}, 6 \mathrm{H}), 0.92(\mathrm{~s}, 6 \mathrm{H}), 0.97(\mathrm{~s}, 6 \mathrm{H}), 2.98$ $(\mathrm{mc}, 4 \mathrm{H}), 3.46(\mathrm{~s}, 3 \mathrm{H}), 7.17-7.35(\mathrm{~m}, 5 \mathrm{H}) .-{ }^{13} \mathrm{C} \mathrm{NMR}\left(\mathrm{CDCl}_{3}\right) \delta 21.55(\mathrm{q}), 23.71(\mathrm{q}), 23.79(\mathrm{q}), 26.25(\mathrm{t})$, $31.79(\mathrm{t}), 46.98(\mathrm{~s}), 48.91(\mathrm{~s}), 49.04(\mathrm{~s}), 51.30$ (q), $125.71(\mathrm{~s}), 126.56$ (d), 127.17 (d), 128.14 (d), 134.34 (s), 134.84 $(\mathrm{s}), 142.97(\mathrm{~s}), 143.76(\mathrm{~s}), 170.09(\mathrm{~s}) .-\mathrm{MS}(70 \mathrm{eV}) \mathrm{m} / \mathrm{z}=338\left(\mathrm{M}^{+}, 67\right), 323(100), 307(13), 306(8), 305(12), 291$ (75), 263 (53), 261 (26), 254 (77), 245 (43), 222 (91), 195 (83). - Anal. Calcd for $\mathrm{C}_{23} \mathrm{H}_{30} \mathrm{O}_{2}$ (338.5): C, 81.61; $\mathrm{H}$, 8.93. Found: $\mathrm{C}, 81.77 ; \mathrm{H}, 8.88$. Traces of an isomer of $6 \mathbf{a}$ (probably double bond migration) were detected in the mother liquors of $6 a$.

3-Methoxycarbonyl-4-phenyl-bicyclo[4.3.0]nona-1(6),3-diene (6b). Compounds $1 \mathrm{~b}(0.205 \mathrm{~g}, 2.17 \mathrm{mmol})$ and 3 $(0.210 \mathrm{~g}, 1.28 \mathrm{mmol})$ were mixed, kept at ambient temperature for $4 \mathrm{~d}$ and worked up as described for $6 \mathrm{a}$ to give $0.339 \mathrm{~g}$ of an oil, which was purified by Kugelrohr distillation $\left(160^{\circ} \mathrm{C} / 0.3 \mathrm{mbar}\right): 0.178 \mathrm{~g}(55 \%)$ of $6 \mathrm{~b}$ which was contaminated by isomers with different location of the double bonds (vinylic protons). $6 \mathrm{~b}$ (tentative assignment): ${ }^{1} \mathrm{H} \mathrm{NMR}\left(\mathrm{CDCl}_{3}, 90 \mathrm{MHz}\right) \delta 1.65-2.15(\mathrm{~m}, \sim 2 \mathrm{H}), 2.2-2.6(\mathrm{~m}, \sim 4 \mathrm{H}), 3.07$ (br. s, $\left.4 \mathrm{H}\right), 3.45(\mathrm{~s}, 3 \mathrm{H}), 7.05-7.45$ $(\mathrm{m}, 5 \mathrm{H}) .-{ }^{13} \mathrm{C} \mathrm{NMR}\left(\mathrm{CDCl}_{3}\right) \delta 21.80(\mathrm{t}), 29.82(\mathrm{t}), 34.93(\mathrm{t}), 35.04(\mathrm{t}), 35.51(\mathrm{t}), 51.31(\mathrm{q}), 125.61(\mathrm{~s}), 126.51(\mathrm{~d})$, 127.10 (d), 128.11 (d), 130.88 (s), 131.51 (s), 142.87 (s), 144.03 (s), 169.80 (s).

trans-3,4-Bis(methoxycarbonyl)-7,7,8,8,9,9-hexamethyl-bicyclo[4.3.0]nona-1(6)-ene (7a). Diene 1a (0.790 g, $4.43 \mathrm{mmol})$ and fumarate $4(0.511 \mathrm{~g}, 3.55 \mathrm{mmol})$ were dissolved in toluene $(2 \mathrm{~mL})$ and heated in a nitrogen armosphere at $80^{\circ} \mathrm{C}$ for $6 \mathrm{~h}$. The ${ }^{1} \mathrm{H}$ NMR spectrum taken after evaporation of excess diene and toluene indicated the quantitative formation of $7 \mathrm{a}$, which was recrystallized from methanol: $\mathrm{Mp} 56-57^{\circ} \mathrm{C}$. IR (KBr): 2916 (broad), $1724,1437,1373,1365,1318,1296,1228,1187,1167,1147,1041 \mathrm{~cm}^{-1}$. - ${ }^{1} \mathrm{H}$ NMR $\left(\mathrm{CDCl}_{3}\right) \delta 0.78(\mathrm{~s}, 6 \mathrm{H}), 0.88$ $(\mathrm{s}, 6 \mathrm{H}), 0.89(\mathrm{~s}, 6 \mathrm{H}), 1.73-2.36(\mathrm{~m}, 4 \mathrm{H}), 2.81-3.05(\mathrm{~m}, 2 \mathrm{H}), 3.71(\mathrm{~s}, 6 \mathrm{H}) .{ }^{13} \mathrm{C} \mathrm{NMR}\left(\mathrm{CDCl}_{3}\right) \delta 21.49(\mathrm{q})$, 23.88 (q), 23.95 (q), 24.97 (t), 42.32 (d), 46.95 (s), 48.94 (s, double intensity), 51.89 (q), 136.44 (s), 175.80 (s). MS (70eV) $m / z=322\left(\mathrm{M}^{+}, 12\right), 307$ (100), 275 (14), 247 (44), 163 (8). - Anal. Calcd for $\mathrm{C}_{19} \mathrm{H}_{30} \mathrm{O}_{4}(322.4)$ : $\mathrm{C}$, 70.77; H, 9.38. Found: C, $70.45 ; \mathrm{H}, 9.52$.

trans-3,4-Bis(methoxycarbonyl)-bicyclo[4.3.0]nona-1(6)-ene (7b). Diene Ib $(0.140 \mathrm{~g}, 1.49 \mathrm{mmol})$ and fumarate $4(0.215 \mathrm{~g}, 1.49 \mathrm{mmol})$ were dissolved in $1.5 \mathrm{~mL}$ of toluene, and heated in a septum-equipped vial, which had been flushed with $\mathrm{N}_{2}$, for $7 \mathrm{~h}$ at $60^{\circ} \mathrm{C}$. The residue obtained after evaporation of toluene was crystallized from methanol to give $0.321 \mathrm{~g}(90 \%)$ of colorless crystals with mp $111-112^{\circ} \mathrm{C}$. - IR (KBr): 2908 ,

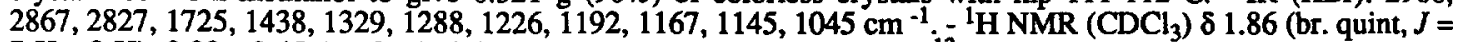
$7 \mathrm{~Hz}, 2 \mathrm{H}), 2.03-2.45(\mathrm{~m}, 8 \mathrm{H}), 2.87-2.95(\mathrm{~m}, 2 \mathrm{H}), 3.70(\mathrm{~s}, 6 \mathrm{H}) .-{ }^{13} \mathrm{C} \mathrm{NMR}\left(\mathrm{CDCl}_{3}\right) \& 22.03(\mathrm{t}), 28.64(\mathrm{t})$, $35.37(\mathrm{t}), 42.20$ (d), $51.87(\mathrm{q}), 132.61(\mathrm{~s}), 175.59(\mathrm{~s})$. $-\mathrm{MS}(70 \mathrm{eV}) \mathrm{m} / \mathrm{z}=238\left(\mathrm{M}^{+}, 21 \%\right), 207(20), 206(17), 178$ (79), 119 (100), 118 (23), 117 (12), 91 (41). - High resolution. MS: $\mathrm{M}^{+}$calcd for $\mathrm{C}_{13} \mathrm{H}_{18} \mathrm{O}_{4} 238.12051$, found 238.12079. 
Competition Experiments. The dienes 1a and 1b were dissolved in ether or toluene, and one of the dienophiles 2,3 , or 4 was added. After completion of the cycloaddition (times see Table), the cycloadduct ratio was determined by GC (Fused silica capillary column SE 30 , length $50 \mathrm{~m}$, i.d. $0.2 \mathrm{~mm}, \mathrm{~N}_{2}$ carrier, $270^{\circ} \mathrm{C}$ column temperature), and the competition constants were evaluated using the formula $k_{d} / k_{b}=\lg \left(1-[D P]_{0} R /[1 \mathrm{a}]_{\mathrm{o}}\right.$ $(1+R)) / \lg \left(1-[D P]_{0} /[1 \mathrm{~b}]_{0}(1+R)\right)$ with $[D P]_{0}=$ initial dienophile concentration and $R=$ molar ratio of the cycloadducts from $1 \mathrm{a}$ and $1 \mathrm{~b} .{ }^{14}$

Table. Determination of the Relative Reactivities of $1 \mathrm{a}$ and $1 \mathrm{~b}$ towards the Dienophiles 2-4.

\begin{tabular}{|c|c|c|c|c|c|}
\hline $\begin{array}{c}\text { Dienophile } \\
\text { (mg) }\end{array}$ & $\underset{(\mathbf{m g})}{\mathbf{1 a}}$ & $\underset{(\mathrm{mg})}{\mathbf{1 b}}$ & $\begin{array}{l}\text { Reaction } \\
\text { conditions }\end{array}$ & $\begin{array}{l}\text { Cycloadducts } \\
\text { (Molar ratio) }\end{array}$ & $k_{a} / k_{b}$ \\
\hline $2(13.8)$ & 64.2 & 37.8 & $\begin{array}{l}0.38 \mathrm{~mL} \mathrm{Et}_{2} \mathrm{O} \\
26 \mathrm{~h}, 20^{\circ} \mathrm{C}\end{array}$ & $5 \mathrm{a}: 5 \mathrm{~b}=5.62$ & 6.98 \\
\hline $2(18.5)$ & 274 & 39.9 & $\begin{array}{l}0.75 \mathrm{~mL} \mathrm{Et}_{2} \mathrm{O} \\
26 \mathrm{~h}, 20^{\circ} \mathrm{C}\end{array}$ & $\mathbf{5 a}: \mathbf{5 b}=22.7$ & 6.49 \\
\hline $3(15.4)$ & 82.3 & 38.8 & $\begin{array}{l}0.25 \mathrm{~mL} \mathrm{Et}_{2} \mathrm{O} \\
14 \mathrm{~h}, 20^{\circ} \mathrm{C}\end{array}$ & $6 \mathrm{a}: 6 \mathrm{~b}=4.45$ & 4.26 \\
\hline $3(13.8)$ & 40.3 & 40.1 & $\begin{array}{l}0.25 \mathrm{~mL} \mathrm{Et}_{2} \mathrm{O} \\
14 \mathrm{~h}, 20^{\circ} \mathrm{C}\end{array}$ & $6 \mathbf{6}: 6 b=1.93$ & 4.11 \\
\hline $4(40.4)$ & 264 & 137 & $\begin{array}{l}1.5 \mathrm{~mL} \text { toluene } \\
5 \mathrm{~h}, 60^{\circ} \mathrm{C}\end{array}$ & $7 \mathbf{a}: 7 \mathbf{b}=0.113$ & 0.102 \\
\hline $4(42.9)$ & 247 & 53.4 & $\begin{array}{l}0.75 \mathrm{~mL} \text { toluene } \\
5 \mathrm{~h} .60^{\circ} \mathrm{C}\end{array}$ & $7 \mathbf{a}: 7 \mathbf{b}=0.350$ & 0.116 \\
\hline
\end{tabular}

Acknowledgment. We thank Dr. A. Sawaryn for carrying out the $M M X$ calculations, Mr. W. Hellebrandt for experimental assistance and the Deutsche Forschungsgemeinschaft for financial support.

References

1) Present address: Institute of Fundamental Chemistry, Technical University of Szczecin, Poland.

2) (a) Levitt, L.S., Widing, H.F., Prog. Phys. Org. Chem. 1976, 12, 119. (b) Chapman, N.B., Shorter, J. (Eds.), Advances in Linear Free Energy Relationships Plenum Press, London, 1972. (c) Shorter, J., Correlation Analysis of Organic Reactivity, Research Studies Press (Wiley), Chichester, 1982. (d) Exner, O., Correlation Analysis of Chemical Data, Plenum Press, New York, 1988.

3) Rüchardt, C., Beckhaus, H.-D., Angew. Chem. 1980, 92, 417; Angew. Chem. Int. Ed. Engl. 1980, 19, 429.

4) For a discussion see: Brown, H.C., The Nonclassical Ion Problem with comments by Schleyer, P.v.R., Plenum Press, New York, 1977.

5) Huisgen, R., Angew. Chem. 1963, 75, 742; Angew. Chem. Int. Ed. Engl. 1963, 2, 633.

6) Giese, B., Angew. Chem. 1983, 95, 771; Angew. Chem. Int. Ed. Engl. 1983, 22, 753.

7) Baran, J., Klein, H., Schade, C., Will, E., Koschinsky, R., Bäuml, E., Mayr, H. Tetrahedron 1988, 44, 2181.

8) (a) Mayr, H., Heigl, U.W., Angew. Chem. 1985, 97, 567; Angew. Chem. Int. Ed. Engl. 1985, 24, 579. (b) Baran, J., Mayr, H., J. Am. Chem. Soc. 1987, 109, 6519. (c) Mayr, H., Heigl. U.W., J. Chem. Soc. Chem. Commun. 1987, 1804.

9) (a) Sauer, J., Angew. Chem. 1967, 79, 76; Angew. Chem. Int. Ed. Engl. 1967, 6, 16. (b) Bäuml, E., Mayr, H., J. Org. Chem. 1983, 48, 2600.

10) MMX version by Gilbert, K.E. and Gajewski, J.J., based on MM2 (Allinger-QCPE 395) and MMP1 Pi (Allinger-QCPE 318) modified by K. Steliou.

11) We thank Prof. R. Gleiter, Heidelberg, for determining the photoelectron spectrum of $1 \mathrm{a}$.

12) Scharf, H.-D., Plum, H., Fleischhauer, J., Schleker, W., Chem. Ber, 1979, I12, 862.

13) Sauer, J., Sustmann, R., Angew. Chem. 1980, 92, 773; Angew. Chem. Int. Ed. Engl. 1980, 19, 779.

14) Ingold, C.K., Shaw, F.R., J. Chem. Soc. 1949, 575. 